\title{
La 'nueva' discapacidad mental
}

\section{The 'new' mental disability}

\section{Palabras clave:}

Discapacidad mental, enfermedad mental, reconocimiento legal de la discapacidad, tipos de discapacidad.

\section{Keywords:}

Mental disability, mental illness, legal accreditation of the disability, types of disability.

\section{El reconocimiento legal de la discapacidad mental}

Del texto de la Convención de Naciones Unidas sobre los Derechos de las Personas con Discapacidad de 2006 (en adelante, la Convención) hay un aspecto muy novedoso que está pasando desapercibido. En concreto, se trata de recoger la existencia de la discapacidad mental como un tipo diferenciado dentro de la categoría jurídica de discapacidad, cuestión que ha sido ya acogida por nuestro ordenamiento jurídico en el actual artículo 4.I del Real Decreto Legislativo I/20I3, de 3 de diciembre, por el que se aprueba el Texto Refundido de la Ley General de derechos de las personas con discapacidad y de su inclusión social (en adelante, LGD). Dicho precepto refunde el artículo I.2 de la Ley 5I/2003, de 2 de diciembre, de igualdad de oportunidades, no discriminación y accesibilidad universal de las personas con discapacidad (conocida por su acrónimo LIONDAU), que había sido modificado por la Ley 26/20I I, de I de agosto, de modificaciones normativas para adaptar nuestro Ordenamiento a la Convención de la ONU.

La primera consecuencia de ello es que, por fin, se ha dividido la anterior

\section{Carlos de Fuentes García- Romero de Tejada \\ <carlosdefuentes@yahoo.es>}

Fundación Manantial. Universidad Complutense de Madrid. Doctor en Derecho por la UCM

\section{Para citar:}

De Fuentes, C. (2016): "La 'nueva' discapacidad mental", Revista Española de Discapacidad, 4 (I): 249-255.

Doi: <http://dx.doi.org/IO.5569/23405IO4.04.OI.I $5>$ 
acepción de 'psíquica', establecida en todos los textos normativos españoles comenzando por el artículo 49 de nuestra Constitución española (CE), en sus dos componentes de 'mental' por una parte e 'intelectual' por otra.

Esta importante distinción no es original de la Convención pues ya la Organización Internacional del Trabajo (OIT) lo recogía en 2002 (OIT, 2002). Asimismo, tampoco se trata de la primera ocasión en la que en nuestro Derecho se utiliza esta expresión. En efecto, ya en los artículos 2.2 y 2.4 de la Ley 39/2006, de I 4 de diciembre, de Promoción de la Autonomía Personal y Atención a las personas en situación de dependencia ya se contempló la separación entre discapacidad intelectual o mental aunque no a efectos definitorios como así ocurre en la Convención o en la LGD.

Este pequeño cambio gramatical tiene una importancia y consecuencias nada desdeñables para el campo de la salud mental.

En primer lugar, desde un punto de vista terminológico, la Convención se ha inclinado por una nomenclatura más corta y fácil ("discapacidad mental") que las otras con las que habitualmente se identifica al colectivo: enfermedad mental, trastorno mental o, incluso, discapacidad psiquiátrica, esta última menos usada en España pero frecuente en la literatura científica internacional (ver, por ejemplo, el importante trabajo de Anthony et al., I990).

Si estudiamos las definiciones del Diccionario de la Real Academia española (RAE), la anterior acepción, 'psíquica', hacía referencia al ámbito más general de la 'psique' o alma humana y por ello se había utilizado para englobar, como ya se ha dicho, a las deficiencias tanto intelectuales como a las propias de las personas afectadas por una enfermedad mental. Por su parte, 'mental', según la tercera entrada del término -la psicológica-, se constriñe "al conjunto de actividades y procesos 'psíquicos', sean o no conscientes”. Y psíquico, a su vez, se identifica con 'psicológico', esto es, relativo a "la ciencia que estudia los procesos mentales en personas y animales".
Podía haberse utilizado también la locución "discapacidad psiquiátrica” pues, según la RAE, se puntualiza como "perteneciente a la ciencia que trata de las enfermedades mentales". No obstante, es un adjetivo con una gran carga histórica negativa y entendemos que el legislador, nacional y de la ONU, acierta al no utilizarla pues, como indica la Estrategia Española sobre Discapacidad 20I 2-2020 (en adelante, "la Estrategia”), en su página diez "los prejuicios de la sociedad constituyen en sí mismos una discapacidad”. Y es comúnmente aceptado que con relación a todo lo que rezume a psiquiátrico existe un prejuicio social evidente. Por consiguiente, el término 'mental' consideramos que es el más ajustado para identificar al conjunto de discapacidades provenientes de la problemática de salud mental.

En segundo término, esta modificación legal debe tener un efecto importante para la visibilidad de las personas que sufren una enfermedad mental. Este colectivo es identificado en la propia Estrategia (página 2) como uno de los elementos claves del progresivo incremento de las personas con discapacidad (PCD) en España y en el mundo. Asimismo, la Estrategia (página 27) indica como uno de sus objetivos principales el 'conocimiento' real de la situación en la que viven las PCD en España. Sin duda, la creación de una figura independiente para la "discapacidad mental" coadyuvará a esa finalidad.

Por último, pero no por ello menos importante, es esencial que lo antes posible se diferencie a la 'nueva' discapacidad 'mental' en las estadísticas y datos oficiales para conocer la situación de este colectivo en empleo, educación y formación, pobreza y exclusión social, etc. Se debe comenzar con el propio reconocimiento legal de la discapacidad, Real Decreto I97I/I999, de 23 de diciembre, de procedimiento para el reconocimiento, declaración y calificación del grado de discapacidad (en adelante, RDPD) pues a partir de la Disposición Final tercera del Real Decreto 290/2004, de 20 de febrero, por el que se regulan los enclaves laborales, se estableció una Disposición Adicional Única en el RDPD, posteriormente modificada en septiembre de 
20I2, para que en todos los certificados de discapacidad se hiciera constar, como mención complementaria, el tipo de discapacidad en las categorías de psíquica, física o sensorial, según corresponda. Por tanto, se debería modificar tal tipología para dividir la 'psíquica', en 'mental' e 'intelectual' para de esta manera poder saber cuántas personas con discapacidad mental existen. En efecto, tras la última modificación de septiembre de 20I2, la Disposición Adicional primera del citado reglamento indica que "a instancia de la persona interesada o de quien ostente su representación, se certificará por el organismo competente el tipo o los tipos de deficiencia o deficiencias que determinan el grado de discapacidad reconocida, conforme a la información que conste en el expediente, a los efectos que requiera la acreditación para la que se solicita”. En la certificación que se expida debería constar la discapacidad 'mental' como tipo independiente.

En este objetivo de saber cuántas personas con trastorno mental tienen reconocimiento legal de discapacidad, deben colaborar tanto la Administración General del Estado como las Comunidades Autónomas. Y, sin duda, debe incluirse en el "Perfil de la Discapacidad de España" que, como se indica en la Estrategia (p. 39) se desarrollará con carácter anual para permitir tener "una serie temporal de diversos indicadores útil para la elaboración de las políticas públicas”.

\section{Requisitos del trastorno mental para generar discapacidad}

Sin ánimo de exhaustividad, vamos a desarrollar siquiera sea brevemente qué requisitos debe atesorar un trastorno mental para generar discapacidad pues no todas las personas que sufren una enfermedad mental presentan una discapacidad. Para que así sea, se precisa que la enfermedad sea considerada grave o, utilizando las palabras del Tribunal de Justicia de la Unión Europea en su sentencia de I I de abril de 20I3, asunto Danmark, apartado 47, que provoque una limitación de larga duración.

El concepto grave es un término polisémico que ha tenido una evolución interesante. Es "equivalente al también utilizado en nuestro contexto "trastorno mental severo", provienen de los vocablos anglosajones "Severe Mental Illnes" o del cada vez más utilizado "Severe and Persistent Mental Illnes". Sin embargo, a lo largo de la historia ha habido diferentes denominaciones para el colectivo de personas con trastorno mental grave: enfermo mental crónico, enfermedad mental grave y persistente, enfermo mental severo, personas con discapacidades psiquiátricas de larga evolución, discapacidad psiquiátrica grave, paciente mental crónico, etc. Aunque en las primeras definiciones el término 'crónico' era de uso generalizado, ya a partir de los 90 empezó a cuestionarse su uso por ser una etiqueta asociada al estigma y por las expectativas pesimistas de mejoría que están relacionadas con el término 'crónico' (González, 2OII: 25-33).

En la actualidad se ha consensuado que la gravedad de una enfermedad o trastorno mental está asociada a tres variables: diagnóstico clínico, duración y funcionamiento psicosocial (Blanco, 20IO: 8I). Veamos con cierto detalle cada uno de estos aspectos:

\section{a. Diagnóstico clínico:}

Siguiendo la "Guía de Práctica Clínica de Intervenciones Psicosociales en el Trastorno Mental Grave" y utilizando la nomenclatura de la Organización Mundial de la Salud (OMS) en su Clasificación Internacional de Enfermedades (CIE) que se encuentra en su décima versión y, por ello se conoce por su acrónimo CIE-Io, (OMS, 2004) ${ }^{\mathrm{I}}$, en el concepto de Enfermedad Mental Grave se incluirían los siguientes diagnósticos clínicos

I. Se sigue la nomenclatura de la OMS (CIE-IO); sistema recomendado por la OMS frente a la otra gran obra para los diagnósticos clínicos de la Asociación Americana de Psiquiatría "El Manual Diagnóstico y Estadístico de los Trastornos Mentales (Diagnostic and Statistical Manual of Mental Disorders)", conocido por sus siglas en inglés, DSM. 
(junto al nombre del diagnóstico, se incluye entre paréntesis su correspondiente número en la clasificación CIE-Io):

- Trastornos esquizofrénicos (F20). Dentro de la categoría 'esquizofrenia' se incluyen varios tipos: paranoide, hebefrénica, simple, catatónica y residual (Pellegrini et al., 20I2: 456-458; Rebolledo y Lobato, 2005)

- Trastornos esquizotípicos (F2I);

- Trastornos delirantes persistentes ( $\left.\mathrm{F}_{22}\right)$;

- Trastornos delirantes inducidos ( $\left.\mathrm{F}_{24}\right)$;

- Trastornos esquizoafectivos (F25);

- Otros trastornos psicóticos de origen no orgánico (F28 y F29);

- Trastorno bipolar (F3 I);

- Episodio depresivo grave con síntomas psicóticos $\left(\mathrm{F}_{32.3}\right)$;

- Trastorno depresivo recurrente $\left(\mathrm{F}_{33}\right)$;

- $\quad$ Trastorno obsesivo compulsivo $\left(\mathrm{F}_{42}\right)$;

Respecto a los Trastornos graves de la personalidad (F62) es práctica habitual incluir el trastorno límite, también denominado Borderline, codificado con el número 301.83 del DSM-IV-TR (González, 20 I I: 30; López y Moreno, 20I 2: 479-499; De Flores et al, 2007).

\section{b. Duración:}

Dentro del concepto de enfermedad mental grave se incluyen aquellas personas que, además de padecer una patología con los diagnósticos antedichos, lleven con la misma una duración prolongada en el tiempo. Siguiendo a Blanco de la Calle "es aquel que progresa o persiste durante un período de tiempo prolongado; en general, toda la vida. Es un término que se contrapone al de 'agudo'" (Blanco, 20Iо: 78). Se han utilizado diferentes criterios para operativizar este criterio. En la actualidad, los más consensuados serían o bien la duración del

En la actualidad, se encuentra en la cuarta edición revisada (DSM-IV-TR) y ya se está elaborando la quinta edición.

El CIE-Io contiene un total de roo categorías (clasificadas de la Aoo a la Z99), que agrupan 329 entidades clínicas individuales. El capítulo V (designado con la letra F) se refiere a los trastornos mentales y del comportamiento y consta de once grandes secciones. tratamiento psiquiátrico (la persona ha de llevar dos o más años en tratamiento en su Centro de Salud Mental), o bien que presente un deterioro importante y progresivo en el funcionamiento psicosocial en los últimos seis meses. Este es el criterio del Grupo de Trabajo de la Guía Clínica de Intervenciones Psicosociales en el Trastorno mental (González, 20I I: 3I).

\section{c. Funcionamiento psicosocial:}

El concepto de enfermedad mental grave, además de definirse desde los criterios de diagnóstico y temporal, se formula desde el prisma del funcionamiento. Este fue un avance apuntado desde los tratamientos de rehabilitación que procuraban a la persona con discapacidad mental desde equipos multiprofesionales sociales (compuesto por psicólogos, trabajadores sociales, terapeutas ocupacionales, educadores sociales, técnicos de empleo, etc.), complementariamente al tratamiento médico y farmacológico prescrito por los equipos de los centros de salud mental (Blanco, 20I0: 79). En efecto, las personas que la padecen tienen graves y duraderas limitaciones para afrontar las demandas de la vida diaria. Se han utilizado diferentes pautas para concretar esta dimensión. Para González, por ejemplo, se concretaría en "recibir prestaciones económicas por la discapacidad, necesitar ayuda en el uso de servicios, dificultades en las actividades de la vida diaria, dependencia de cuidadores o servicios, dificultades en el funcionamiento social, dificultades en el funcionamiento laboral, alta vulnerabilidad al estrés, etc." (González, 2OII: 3I-32). No obstante, existe consenso para determinar que las personas con trastorno mental grave suelen presentar déficit, con mayor o menor grado de afectación, en una o varias de las siguientes áreas de funcionamiento psicosocial (Blanco, 2010: 89-90):

- Autocuidados: problemas con la higiene personal, hábitos de vida no saludables, etc.

- Autonomía: deficiente manejo del dinero, dificultades en el manejo de transportes, dependencia económica y mal desempeño laboral. 
- Autocontrol: apuros para manejar situaciones de estrés, falta de competencia personal, etc.

- Relaciones interpersonales: carencia de red social, inadecuado manejo de situaciones sociales, déficit en habilidades sociales.

- Ocio y tiempo libre: aislamiento, conflicto para manejar el ocio, limitaciones para disfrutar, falta de motivación e interés.
Por tanto, resumiendo todo lo dicho con los tres criterios expuestos, la enfermedad mental grave "designa al conjunto de personas que sufren entidades clínicas diferentes pero que además evidencian una serie de problemas comunes que se expresan a través de diferentes discapacidades" (Blanco, 20IO: 8I) que nosotros aglutinamos en el macro concepto de discapacidad mental pues como afirman Els et al., la discapacidad mental no se puede basar únicamente en el diagnóstico clínico (Els et al., 20I2: 344-345). 
Referencias bibliográficas

Anthony, W. et al. (1990): Psychiatric rehabilitation, Boston: Center for Psychiatric Rehabilitation.

Blanco de la Calle, A. (20I0): "El enfermo mental con discapacidades psicosociales", en Pastor, A. et al. (Coords.): Manual de rehabilitación del trastorno mental grave. Madrid: Editorial Síntesis.

De Flores, T. et al. (2007): Trastorno límite de la personalidad a la búsqueda del equilibrio emocional. Una guía para profesionales, familias y pacientes, Madrid: Morales y Torres Editores.

Els, C. et al. (2OI2): "Workplace Functional Impairment Due to Mental Disorders", en Mental Illnesses - Understanding, Prediction and Control. Prof. Luciano LAbate. Editorial InTech (en línea). <http://www.intechopen. com/books/mental-illnessesunderstandingprediction-and-control/workplace-functionalimpairment-due-to-mental-disorders $>$, acceso I 8 de febrero de 2016.

España. Ley 26/20I I, de I de agosto, de adaptación normativa a la Convención Internacional sobre los Derechos de las Personas con Discapacidad, Boletín Oficial del Estado, 2 de agosto de 20 I I, núm. I 84 , pp. 87.478-87.494.

España. Ley 5 I/2003, de 2 de diciembre, de igualdad de oportunidades, no discriminación y accesibilidad universal de las personas con discapacidad, Boletín Oficial del Estado, 3 de diciembre de 2003 , núm. 289 , pp. 43.I87-43.195.

España. Real Decreto I97I/I999, de 23 de diciembre, de procedimiento para el reconocimiento, declaración y calificación del grado de discapacidad, Boletín Oficial del Estado, 26 de enero de 2000, núm. 22, Correcciónta de errores, Boletín Oficial del Estado, núm. 62, de I3 de marzo.

España. Real Decreto-Legislativo I/20I3, de 29 de noviembre, por el que se aprueba el Texto
Refundido de la Ley General de derechos de las personas con discapacidad y de su inclusión social, Boletín Oficial del Estado, 3 de diciembre de 2013 , núm. 289 , pp. $95.635-95.673$.

Estrategia Española sobre Discapacidad 20I 22020. (20II). Accesible en: <http://sid.usal.es/ libros/Discapacidad/26 I I 2/8-4-I/estrategiaespanola-sobre-Discapacidad-20 I 2-2020. aspx>, acceso I 8 de febrero de 2016.

González-Cases, J. C. (20I I): Violencia en la pareja hacia mujeres con trastorno mental grave, Madrid: Universidad de Alcalá, Tesis Doctoral, Departamento de Especialidades Médicas.

Grupo de Trabajo de la Guía de Práctica Clínica de Intervenciones Psicosociales en el Trastorno Mental Grave (2009): Guía de Práctica Clínica de Intervenciones Psicosociales en el Trastorno Mental Grave,Madrid: Plan de Calidad para el Sistema Nacional de Salud del Ministerio de Sanidad y Política Social.

López, A. y Moreno, E. (20I2): "Desarrollo profesional e inserción laboral en personas con trastornos de la personalidad", en Sánchez, O. (coord.): Desarrollo profesional e inserción laboral en personas con enfermedad mental, Madrid: Editorial Grupo 5.

Organización de Naciones Unidas. Convención de Naciones Unidas sobre los Derechos de las Personas con Discapacidad de I 3 de diciembre de 2006, Resolución publicada el 24 de enero de 2007. Ratificada por España por medio de Instrumento de ratificación, Boletín Oficial del Estado, 2I de abril de 2008, núm. 96, pp. 20.648-20.659.

Organización Internacional del Trabajo,OIT (2002): Gestión de las Discapacidades en el lugar de trabajo. Repertorio de recomendaciones prácticas de la OIT, Ginebra: Oficina Internacional del Trabajo (en línea) $<$ http://www.ilo.org/wcmsp 5/groups/public/--ed_protect/---protrav/---safework/documents/ 
normativeinstrument/wcms_2 I 8554.pdf>, acceso 26 de abril de 2016.

Organización Mundial de la Salud, OMS (2004): Clasificación Internacional de las Enfermedades. Io revisión (en línea). $<$ http://apps.who.int/classifications/apps/icd/ icd Ioonline2004/fr-icd.htm>, acceso 26 de abril de 2016.

Pellegrini Spangenberg, M. et al. (2OI2): "Desarrollo profesional en personas con trastornos psicóticos”, en Sánchez, O. (coord.):
Desarrollo profesional e inserción laboral en personas con enfermedad mental. Madrid: Editorial Grupo 5.

Real Academia Española, RAE (200I): Diccionario de la RAE, $22^{a}$ edición Espasa Calpe, $5^{\mathrm{a}}$ actualización de 20 I I (en línea). <http://lema. rae.es/drae200I/>, acceso 26 de abril de 2016 .

Rebolledo Moller, S. y Lobato Rodríguez, M.J. (2005): Cómo afrontar la esquizofrenia. Una guía para familiares, cuidadores y personas afectadas, Madrid: Grupo Aula Médica. 